\title{
APPLICATIONS TO ANALYSIS OF A TOPOLOGICAL DEFINITION OF SMALLNESS OF A SET
}

\author{
BY J. L. DOOB
}

1. Introduction. In this paper a certain definition of smallness of a set in a topological space is applied to various problems in measure theory and potential theory. The small sets are necessarily countable if the space has a countable basis but the topological spaces in the applications will not have countable bases, and in fact the main point in the applications will be to verify that these spaces have a much weaker property, a weakening of the Lindelöf property: an arbitrary union of open sets is to be equal to a countable subunion, up to a small set. This property and its implications are discussed in the next section. In $\$ 3$ an abstract mechanism is set up to facilitate proofs that a given topological space has a weak Lindelöf property. In $\$ 4$ it is shown how any Radon measure $\nu$ on Euclidean space determines a topology on the space, going back, if the measure is Lebesgue measure and the dimensionality 1 , to Denjoy's discussion of approximately continuous functions. It is shown that in this topology Euclidean space has a weak Lindelöf property. In this application the class of small sets is the class of sets of $\nu$ measure 0 . After a review of the fine topology of potential theory from a point of view which illustrates the role of this topology in the fundamental convergence theorem of potential theory and in the usual smoothing operations, it is shown that in a rather general probabilistic framework the space of probabilistic potential theory, in the fine topology, has a weak Lindelöf property. The small sets in the classical case are the polar sets, in the general case are the semipolar sets, under some restrictions on the probabilistic context. The general theorems on lower envelopes of various classes of upper semicontinuous functions on any space with a weak Lindelöf property yield new results and unify and generalize old ones.

In all applications, a weakened Lindelöf property makes it possible to reduce certain problems involving lower envelopes of families of upper semicontinuous functions, in particular (by way of indicator functions) intersections of families of closed sets, to problems involving countable families. For example, in the specialization to classical potential theory, among other results it is shown that if $A \rightarrow \gamma(A)$ is a function from subsets of the given space to the extended reals, with

A Presidential Address delivered before the Chicago meeting of the Society on January 26, 1966. 
certain properties enjoyed for example by the capacity of $A$, and $\left\{F_{t},(t \in T)\right\}$ is a decreasingly directed class of fine closed sets, then $\gamma\left(\cap_{t} F_{t}\right)=\inf _{t} \gamma\left(F_{t}\right)$. References to related work of Choquet, Getoor, Brelot will be made below.

2. The quasiLindelöf property. Let $R$ be a Hausdorff space. All functions on $R$ will be to the extended reals. If $u$ is any such function, define $u^{\prime}$ as the limit superior function of $u$, that is, the function defined by

$$
u^{\prime}(\xi)=\limsup _{\eta \rightarrow \xi} u(\eta)
$$

except that if $\xi$ is an isolated point of $R, u^{\prime}(\xi)=u(\xi)$. (The limit concept used here does not involve the value of the function at the point of approach.) The function $u$ is upper semicontinuous if and only if $u \geqq u^{\prime}$. For any function $u, u^{\prime}$ is upper semicontinuous. If $\rho$ is a countable ordinal $u^{(\rho)}$ is defined as $u^{\prime}$ if $\rho=1$ and if $u^{(\sigma)}$ is defined for $\sigma<\rho, u^{(\rho)}$ is defined as $\left(u^{(\rho-1)}\right)^{\prime}$ if $\rho$ is not a limit ordinal, as $\lim _{\sigma \rightarrow \rho} u(\sigma)$ if $\rho$ is a limit ordinal. If $A$ is a subset of $R, A^{\prime}$ is defined as the union of the set of points of $A$ which are isolated points of $R$ and the set of limit points of $A$, so that if $I_{B}$ is the indicator function of a set $B$, $I_{B^{\prime}}=\left(I_{B}\right)^{\prime} ; A^{(\rho)}$ is then defined by transfinite induction in the obvious way for $\rho$ a countable ordinal or equivalently defined as the set for which $I_{A}^{(p)}=\left(I_{A}\right)^{(p)}$. We shall use the fact, easily proved by transfinite induction, that for upper semicontinuous $u$ and every countable ordinal $\rho$,

$$
\{\xi: u(\xi) \geqq r\}^{(\rho)} \subset\left\{\xi: u^{(\rho)}(\xi) \geqq r\right\} \subset\{\xi: u(\xi) \geqq s\}^{(\rho)} \text { if } s<r .
$$

Definition. $A$ subset of $R$ will be called weakly quasinull if there is an upper semicontinuous function $u$ such that $u>u^{\prime}$ on $A$; if $u^{\prime}$ is continuous $A$ will be called quasinull. $A$ property will be said to hold [weakly] quasieverywhere if it holds except on a [weakly] quasinull set.

Every quasinull set is weakly quasinull and a subset of a set of either type is of the same type. A set of either type contains no isolated point of $R$. It is no restriction to suppose that $u$ in the definition just given has its range in an arbitrary interval. The following assertions contain useful further details.

(a) A countable union of quasinull or weakly quasinull sets is of the same type. To see this choose $u_{n}$ upper semicontinuous in such a way that $u_{n}>u_{n}^{\prime}$ on the $n$th set, and that $0 \leqq u_{n} \leqq 2^{-n}$. Then if $u=\sum u_{r}$, $u>u^{\prime}$ on the union of the sets and $u^{\prime}$ is continuous if each $u_{n}^{\prime}$ is.

(b) For arbitrary $u$ on $R$, the set where $u>u^{\prime}$ is weakly quasinull and 
is quasinull if $u^{\prime}$ is continuous. To see this let $v=\max \left[u, u^{\prime}\right]$. Then $v^{\prime}=u^{\prime}$ and $v$ is upper semicontinuous. Moreover

$$
\left\{\xi: u(\xi)>u^{\prime}(\xi)\right\}=\left\{\xi: v(\xi)>v^{\prime}(\xi)\right\}
$$

so that the set on the left is weakly quasinull, quasinull if $u^{\prime}$ is continuous, as asserted. In particular, applying the assertion to indicator functions of sets, if $A$ is an arbitrary set $A-A \cap A^{\prime}$ is weakly quasinull, and is quasinull if $A^{\prime}$ is empty.

(c) A set is weakly quasinull [quasinull] if and only if it contains no isolated point of $R$ and is a countable union of isolated sets [sets with no limit points]. In fact by (a) and (b) a set with the stated property is weakly quasinull or quasinull. Conversely suppose that $A$ is weakly quasinull, say

$$
A \subset\left\{\xi: u(\xi)>u^{\prime}(\xi)\right\}
$$

where $u$ is upper semicontinuous. Then $A$ contains no isolated point of $R$ and

$$
A=\underset{a, b}{\bigcup} A \cap\left\{\xi: u(\xi) \geqq b>a \geqq u^{\prime}(\xi)\right\}
$$

where $a, b$ run through the rationals. Each brace is an isolated set so $A$ is a countable union of isolated sets. If $A$ is even quasinull we can suppose that $u^{\prime}$ is continuous and in this case each brace is a set with no limit points, so $A$ is a countable union of such sets.

Note that if $B \cap B^{\prime}=\varnothing, B=C-C^{\prime}$, where $C=B \cup B^{\prime}$, so that every weakly quasinull set can be expressed as a countable union of sets of the form $C-C^{\prime}$ with $C$ closed.

Definition. $A$ topological space will be said to have the [weak] quasiLindelöf property if every union of open sets is equal, up to a [weakly] quasinull set to a countable subunion. If the discrepancy can be made a set $A$ with $\left[A \cap A^{(n)}=\varnothing\right] A^{(n)}=\varnothing$, the space will be said to have the [weak] quasiLindelöf(n) property.

The index $n$ in this definition can be any countable ordinal.

If a space has the [weak] quasiLindelöf ${ }^{(m)}$ property it has the [weak] quasiLindelöf ${ }^{(n)}$ property for $n>m$ and the [weak] quasiLindelöf property. A trivial covering argument shows that if $R$ has the quasiLindelöf property a weakly quasinull set is the union of a countable set containing no isolated points of $R$ and a quasinull set. It follows that if $R$ has the quasiLindelöf property every weakly quasinull set is quasinull.

Lemma 2.1. A space has the weak quasiLindelöf(n) property if and only if whenever $F$ is the intersection of a family of closed sets there is an intersection $F_{\infty}$ of a countable subfamily such that $F_{\infty}^{(n)} \subset F \subset F_{\infty}$. 
The relation $F \subset F_{\infty}$ is of course trivial: only the left half of the inclusion relation is significant. The dual version of this result, obtained by complementation, makes it clearer that this lemma sharpens the weak quasiLindelö ${ }^{(n)}$ property: according to the lemma a space has the weak quasiLindelöf ${ }^{(n)}$ property if and only if whenever $G$ is the union of a family of open sets there is a union $G_{\infty}$ (the complement of $F_{\infty}$ ) of a countable subfamily such that $G_{\infty} \subset G C^{(n)} G_{\infty}$. Here ${ }^{(1)} H$ for any open set $H$ is the union of $H$ with those isolated points of its complement which are not isolated points of the space, ${ }^{(2)} H={ }^{(1)}\left({ }^{(1)} H\right)$ and so on. The condition of the lemma is sufficient because under this condition if $A=G-G_{\infty}=F_{\infty}-F$ it follows that

$$
A \cap A^{(n)} \subset A \cap F_{\infty}^{(n)} \subset A \cap F=\varnothing .
$$

To prove that the condition of the lemma is necessary suppose that the space has the weak quasiLindelöf ${ }^{(n)}$ property, so that there is a countable intersection $F_{\infty}$ with $\left(F_{\infty}-F\right) \cap\left(F_{\infty}-F\right)^{(n)}=\varnothing$. Then, denoting complementation by $\sim$,

$$
F_{\infty}^{(n)} \subset\left(F_{\infty}-F\right)^{(n)} \cup F^{(n)} \subset\left(F_{\infty}-F\right)^{\sim} \cup F^{(n)} \subset \tilde{F}_{\infty} \cup F
$$

so $F_{\infty}^{(n)} \subset F$ (because $F_{\infty}^{(n)} \cap \widetilde{F}_{\infty}=\varnothing$ ) as was to be proved.

If the space has the quasiLindelöf ${ }^{(n)}$ property, $F_{\infty}$ can be chosen as stated in the lemma and in addition so that $\left(F_{\infty}-F\right)^{(n)}=\varnothing$. The latter relation implies (but is not implied by) the equality $F_{\infty}^{(n)}=F^{(n)}$.

THEOREM 2.1. A space has the weak quasiLindelöf $f^{(n)}$ property if and only if whenever $u$ is the lower envelope of a family of upper semicontinuous functions there is a countable subfamily with lower envelope $u_{\infty}$ such that $u_{\infty}^{(n)} \leqq u \leqq u_{\infty}$. A space has the [weak] quasiLindelöf property if and only if when $u$ is as above there is a countable subfamily with lower envelope $u_{\infty}$ such that $u_{\infty}=u$ [weakly] quasieverywhere.

Note that $u_{\infty}=u_{\infty}^{(n)}$ weakly quasieverywhere, quasieverywhere if the space has the quasiLindelöf property. Since an upper semicontinuous function $f$ on a completely regular space is the lower envelope of the class of continuous functions $\geqq f$, Theorem 2.1 implies that if $R$ is completely regular and has the [weak] quasiLindelöf property every upper semicontinuous function is the [weakly] quasieverywhere limit of a decreasing sequence of continuous functions.

If the functions in the theorem are indicator functions of closed sets the first part of the theorem reduces to the lemma, so it is sufficient to prove that the condition of the lemma implies that of the theorem. Suppose then that a family of upper semicontinuous func- 
tions is given, with lower envelope $u$. Applying the lemma, choose a countable subfamily, whose lower envelope $u_{\infty}$ satisfies the condition

$$
\left\{\xi: u_{\infty}(\xi) \geqq s\right\}^{(n)} \subset\{\xi: u(\xi) \geqq s\}
$$

for all rational $s$. It is sufficient to prove that $u_{\infty}^{(n)} \leqq u$. Now according to (2.1)

$$
\left\{\xi: u_{\infty}^{(n)}(\xi) \geqq r\right\} \subset\left\{\xi: u_{\infty}(\xi) \geqq s\right\}^{(n)} \text { if } s<r .
$$

If $u_{\infty}^{(n)}(\xi)=\alpha$, and if $r<\alpha, \xi$ is in the set on the left side of (2.3), therefore in the set on the right side of $(2.2)$ for $s<r$. Hence $u(\xi) \geqq \alpha$, so $u_{\infty}^{(n)} \leqq u$, as was to be proved. If the space has the [weak] quasiLindelöf property and if $u$ is the lower envelope of a family of upper semicontinuous functions then, using the complementary version of the quasiLindelöf property, there is a countable subfamily, with lower envelope $u_{\infty}$, such that simultaneously for all rational $r$ the difference

$$
\left\{\xi: u_{\infty}(\xi) \geqq r\right\}-\{\xi: u(\xi) \geqq r\}
$$

is [weakly] quasinull. Neglecting a [weakly] quasinull set this difference is empty simultaneously for all real $r$, so $u_{\infty}=u$ off this [weakly] quasinull set. Conversely if the condition of the second part of the theorem is satisfied (even only for indicator functions of closed sets) it is obvious that $R$ has the [weak] quasiLindelöf property.

If the space has the quasiLindelöf ${ }^{(n)}$ property, $u_{\infty}$ can be chosen as in the theorem and in addition so that $u_{\infty}^{(n)}=u^{(n)}$, but the validity of this condition is not sufficient to yield the quasiLindelöf ${ }^{(n)}$ property.

The class $B$ of Borel subsets of $R$ is defined as the Borel field generated by the class of closed sets. Since every weak quasinull set is a countable union of sets $A-A^{\prime}$ with $A$ closed, weakly quasinull sets are Borel sets.

Definition. If $\gamma$ is an increasing function from a class of Borel measurable functions to the extended reals, $\gamma$ will be called a [weak] quasicapacity if the following conditions are satisfied.

(i) If $u_{1}>u_{2}>\cdots$ are upper semicontinuous functions in the domain of $\gamma, u=\lim _{n \rightarrow \infty} u_{n}$ is in the domain, and $\gamma(u)=\lim _{n \rightarrow \infty} \gamma\left(u_{n}\right)$ if the latter limit is not $+\infty$.

(ii) If $u$ is in the domain of $\gamma$ and if $v \leqq u$ with equality [weakly] quasieverywhere then $v$ is in the domain and $\gamma(u)=\gamma(v)$.

If the space has the quasiLindelöf property a quasicapacity is a weak quasicapacity. If the domain of $\gamma$ is a class of indicator func- 
tions of sets we shall write $\gamma(A)$ instead of $\gamma\left(I_{A}\right)$. Every measure on $B$ which vanishes on [weakly] quasinull sets is a [weak] quasicapacity.

THEOREM 2.2. If $R$ has the [weak] quasiLindelöf property, if $\left.\left\{u_{t}, t \in T\right)\right\}$ is a generalized decreasing sequence (=directed decreasing family) of upper semicontinuous functions with limit $u$, and if every $u_{t}$ is in the domain of a [weak] quasicapacity $\gamma$ then $u$ is in the domain of $\gamma$, and $\gamma(u)=\lim _{t} \gamma\left(u_{t}\right)$ whenever the limit is not $+\infty$.

In fact the quasiLindelöf property (or weak one, as the case may be) reduces this theorem to the case of ordinary sequences, for which the desired property is part of the definition of a quasicapacity.

Let $\mu$ be a measure whose domain includes $B$ and suppose that $\mu=\sum \mu_{n}$ is the sum of countably many finite measures. Then there is a weakly quasinull set $A_{0}$ such that $\mu$ vanishes on weakly quasinull subsets of $R-A_{0}$. $\left(\mu_{n}\left(A_{0}\right)\right.$ maximizes $\mu_{n}(B)$ for $B$ weakly quasinull.) With this definition of $A_{0}, \mu$ can be written as the sum of two measures,

$$
\mu(A)=\mu\left(A \cap\left(R-A_{0}\right)\right)+\mu\left(A \cap A_{0}\right),
$$

the first vanishing on every weakly quasinull set, the second supported by a weakly quasinull set. The decomposition is unique, although $A_{0}$ is not. There is a corresponding decomposition if "weakly" is omitted throughout. The following theorem shows that the first component has a minimal closed support, uniquely determined by $\mu$ since the first component is.

THEOREM 2.3. Let $R$ have the [weak] quasiLindelöf property and let $\mu$ be a measure on $B$ which is the sum of countably many finite measures and which vanishes on [weakly] quasinull sets. Then there is a smallest closed support $F$ of $\mu$, and $F=F^{\prime}$.

Let $F$ be the intersection of the class of closed supports of $F$. According to Theorem 2.1 if $R$ has the [weak] quasiLindelöf property there is a countable subclass with intersection say $F_{\infty}$ such that $F_{\infty}-F$ is [weakly] quasinull. Since $F_{\infty}$ is a support of $\mu, F$ is also, so $F$ is the smallest closed support. Since $F^{\prime} \subset F$ and the difference is weakly quasinull, quasinull if $R$ has the quasiLindelöf property, $F^{\prime}$ is also a closed support, so $F^{\prime}=F$.

If $\mu$ is replaced by any family of measures this argument shows that there is a smallest closed set which is simultaneously a support for every measure in the family. 
ThEOREM 2.4. Suppose that $R$ has the [weak] quasiLindelof property, let $f_{1}, f_{2}$ be functions from $R$ to the extended reals, with $f_{1} \leqq f_{1}^{\prime}$ and let $\mathfrak{R}$ be a class of subsets of $R$, containing the [weakly] quasinull sets and containing the countable unions of its members. Then the lower envelope $u$ of the class $\Gamma$ of upper semicontinuous functions $\geqq f_{1}$ on $R$, and also $\geqq f_{2}$ neglecting a set in $\mathfrak{N}$, is in $\Gamma$, and $u=u^{\prime}$.

If $v \in \Gamma, v^{\prime} \in \Gamma$ also, because $v \geqq f_{1}$ implies that $v^{\prime} \geqq f_{1}^{\prime} \geqq f_{1}$, and $v \geqq f_{2}$ neglecting an $\mathfrak{N}$ set implies the same for $v^{\prime}$. If the lower envelope $u$ is in $\Gamma, u=u^{\prime}$ because $u^{\prime} \in \Gamma$ and $u^{\prime} \leqq u$. The function $u$ is trivially upper semicontinuous and $\geqq f_{1}$. Let $u_{\infty}$ be the lower envelope of a countable subclass of $\Gamma$ chosen so that $u_{\infty}=u$ [weakly] quasieverywhere. Then $u_{\infty}$ is in $\Gamma$ so $u$ is also, as was to be proved.

Note that Theorem 2.3 is a special case of Theorem 2.4. In fact if $f_{1}=0$ and $f_{2}=1$ and if $\mathfrak{N}$ is the class of sets of $\mu$ measure 0 the minimizing function $u$ of Theorem 2.4 is easily seen to be the indicator function of the minimal closed support of $\mu$.

3. Axioms for lower envelopes. The partial orders used below are supposed transitive and reflexive. If $X$ is a partially ordered set, a subset $A$ may or may not have an order infimum, and there may be more than one. An element will be called a countable infimum of $A$ if it is an infimum both of $A$ and of some countable subset of $A$.

In many contexts the following axioms are satisfied.

$\boldsymbol{A}_{1}$. $\Gamma$ is a distinguished family of functions from a set $R$ to the extended reals; $\Gamma$ contains the lower envelope of every countable subfamily.

$A_{2} . u \rightarrow u^{*}$ is a transformation from $\Gamma$ into the class of functions from $R$ into the extended reals, satisfying the conditions

$$
\begin{aligned}
& u^{*} \leqq u, \\
& u_{1} \leqq u_{2} \text { implies that } u_{1}^{*} \leqq u_{2}^{*} .
\end{aligned}
$$

$\boldsymbol{A}_{3}$. If $\Gamma_{0}$ is a subfamily of $\Gamma$, with lower envelope $u$, there is a countable subfamily of $\Gamma_{0}$ with lower envelope $u_{\infty}$ satisfying $u_{\infty}^{*} \leqq u$ $\leqq u_{\infty}$.

The fact that $u \leqq u_{\infty}$ is trivial even without the axiom. If $\boldsymbol{A}_{3}$ is satisfied whenever $\Gamma_{0}$ is a directed decreasing family it is satisfied for all $\Gamma_{0}$. If the functions involved are all indicator functions of sets we shall write $B^{*}$ for the set whose indicator function is $I_{B}^{*}$ and $\Gamma, \Gamma_{0}$ will be taken as classes of sets.

Theorem 3.1. Let $X$ be a partially ordered space under « and suppose that every directed decreasing subset of $X$ has a countable infimum. 
Let $\hat{X}$ be the space of vectors with some countable number of $X$-valued components. Suppose that $\boldsymbol{A}_{1}$ and $\boldsymbol{A}_{\mathbf{2}}$ are satisfied and that there is an increasing function $\boldsymbol{C}$ from $\Gamma$ into $\hat{X}$ (ordered componentwise) with the property that $C(u) \ll C(v)$ implies that $u^{*} \leqq v^{*}$. Then $A_{3}$ is satisfied.

It is sufficient to prove the theorem for $X=\hat{X}$, that is in the one dimensional case, because in the order described $\hat{X}$ actually has the very properties demanded of $X$. The phraseology of the theorem was chosen to make it easier to apply. In the one dimensional case a directed subfamily $\Gamma_{0}$ of $\Gamma$ maps under $u \rightarrow C(u)$ into a directed subset of $X$. Suppose that $\inf _{n} \mathbf{C}\left(u_{n}\right)$ is a countable infimum of the latter subset, and that $u_{\infty}$ is the lower envelope of the $u_{n}$ sequence. Then $u_{\infty}$ is in $\Gamma$ and $\boldsymbol{C}\left(u_{\infty}\right) \leqq C(v)$ for every $v$ in $\Gamma_{0}$, so $u_{\infty}^{*} \leqq v^{*} \leqq v$ for every $v$ in $\Gamma_{0}$. We conclude that $u_{\infty}^{*} \leqq u$, thus obtaining the nontrivial half of $\boldsymbol{A}_{\mathbf{3}}$.

4. Application to measures on Euclidean space. Let $R$ be an open subset of a Euclidean space and let $\nu$ be a completed Radon measure of subsets of $R$. Define the $\nu$ topology by the convention that $\xi$ is a $\nu$ limit point of a set $A$ not containing $\xi$ if for every $\nu$ measurable superset $B$ of $A$

$$
\lim \sup _{I} \frac{\nu(B \cap I)}{\nu(I)}>0 .
$$

Here $I$ is a member of a family of sets, each containing $\xi$, and the limit superior is taken as the diameter of $I$ shrinks to 0 . For each $\xi$ the family of sets $I$ is to have the property that the fundamental derivative theorem is valid: when $C$ is $\nu$ measurable, $\lim _{I} \nu(C \cap I) / \nu(I)$ $=1$ at $\nu$ almost every point $\xi$ of $C$ when $I$ shrinks to $\xi$ in the prescribed family. We assume that $\nu$ and the $I$ family have the property that, for each $\xi, \nu(I)>0$ for all $I$ in the prescribed family. We thus consider only measures which are strictly positive on nonempty (Euclidean) open subsets of $R$. The $\nu$ isolated points of $R$ are the points of strictly positive $\nu$ measure. For example, if $\nu$ is Lebesgue measure the $I$ family for $\xi$ can be the family of intervals containing $\xi$ and with edges parallel to the coordinate axes. In this case what is involved is the strong derivative [10]. For general $\nu$ (assigning strictly positive measure to nonempty Euclidean open sets) $I$ can range through the balls or cubes with center $\xi$ or more generally through the sets of a Morse star blanket [9].

Specializations and generalizations of this topology have been discussed by many authors since the introduction by Denjoy of approxi- 
mately continuous functions, recently for example by Goffman, Neugebauer and Nishiura [4] and by Zink [11]. Applying the density theorem, $\nu$ almost every point of a set is either a $\nu$ limit point of the set or a $\nu$ isolated point of $R$. If $A$ is $\nu$ measurable its $\nu$ closure and its $\nu$ interior differ from $A$ by sets of $\nu$ measure 0 . The set of $\nu$ limit points of an arbitrary set is the same as that of any $\nu$ measure cover of the set. Thus the $\nu$ closed sets and hence the $\nu$ Borel sets are $\nu$ measurable. Every $\nu$ weakly quasinull set is $\nu$ quasinull and a set is $\nu$ quasinull if and only if it is of $\nu$ measure 0 . Let $u^{\prime}$ be the limit superior function of $u$ in the $\nu$ topology and define $A^{\prime}$ correspondingly for the set $A$.

THEOREM 4.1. The space $R$ in the $\nu$ topology has the quasiLindelöf(1) property.

Since the $\nu$ weakly quasinull sets are $\nu$ quasinull it is sufficient to prove that this property is valid using the criterion of Lemma 2.1. Let $\Gamma=\Gamma_{0}$ be a family of $\nu$ closed sets with intersection $F$. In applying Lemma 2.1 it is no restriction to assume that $\Gamma_{0}$ contains the countable intersections of its members. We shall apply Theorem 3.1 to show that a set $F_{\infty}$ exists as described in the lemma with $n=1$. We use the set version of the axioms $A_{1}, A_{2}, A_{3}$, defining $A^{*}$ as $A^{\prime}$. Let $X$ be the set of extended real numbers with the usual order and if $G$ is a finite union of sets of a countable basis for the topology of $R$ and if $A$ is $\nu$ closed define $\boldsymbol{C}_{G}(A)=\nu(G \cap A)$. If $\boldsymbol{C}_{G}(A) \leqq \boldsymbol{C}_{G}(B)$ for every $G, A^{\prime} \subset B^{\prime}$. Thus the hypotheses of the set version of Theorem 3.1 are satisfied, with $\mathrm{C}(A)$ the vector with components $\left\{\mathbf{C}_{G}(A)\right\}$, as was to be proved. It is instructive to see what changes would be made if the criterion of Theorem 2.1 instead of Lemma 2.1 were to be used. In this application of Theorem $3.1, \Gamma=\Gamma_{0}$ is a family of upper semicontinuous functions, $u^{*}=u^{\prime}, X$ is the space of extended real valued monotone nondecreasing left continuous functions on $[0, \infty)$ ordered by pointwise inequality. If $u$ is in $\Gamma$ and $G$ is as above $C_{G}(u)$ is the element of $X$ which at $s$ has the value $\nu\{\xi: \xi \in G, u(\xi) \geqq s\}$.

As an example of the interpretation in the present context of the theorems in $\$ 2$ we apply Theorem 2.3 , obtaining: if $\mu$ is a Radon measure absolutely continuous with respect to $\nu$ there is a smallest $\nu$ closed set which supports $\mu$. (If $h$ is the Radon-Nikodym derivative $d \mu / d \nu$, this smallest support is the $\nu$ closure of the subset of $R^{\prime}$ where $h$ is strictly positive.) As a second example let $\nu$ be Lebesgue measure and define the $\nu$ topology with $I$ in (4.1) a cube with center $\xi$. Let $u$ be a Lebesgue measurable function. Then it is approximately continuous almost everywhere so it is $\nu$ continuous almost everywhere, hence coincides almost everywhere with a $\nu$ upper semicontinuous function 
$u_{1}$. Goffman, Nishiura and Neugebauer [4] proved that in this topology the space is completely regular and it therefore follows from the quasiLindelöf property (see the remarks after the statement of Theorem 2.1) that $u_{1}$ and therefore also $u$ are the almost everywhere limit of a decreasing sequence of $\nu$ continuous functions. The latter result is due to Zink [11].

5. The fine topology in potential theory-classical case. The definitions in $\$ 2$ were suggested by the properties of the fine topology of potential theory. In the classical case this topology on a Green space (the Green space hypothesis that the space supports a nonconstant positive superharmonic function is unnecessary if minor modifications are made below) is defined as the coarsest making superharmonic functions continuous. Concepts relative to the fine topology will be identified by the qualifier "fine." To avoid trivial special remarks we suppose that $R$ has no points at infinity if the dimensionality exceeds 2. (Such points would have to be treated like isolated points. Since no point of the space is a limit point of the set of those points nothing is lost for our purposes by excluding them.) The fine limit superior function of a function $u$ will be denoted by $u^{\prime}$ and if $A$ is a set $A^{\prime}$ is defined correspondingly. No point of $R$ is fine isolated. A set is polar, that is, of zero capacity if and only if it has no fine limit points, equivalently if and only if it is fine isolated. A countable union of polar sets is polar. Thus the classes of fine weakly quasinull and fine quasinull sets coincide with the class of polar sets. "Fine weakly quasieverywhere" and "fine quasieverywhere" both reduce to "except for a polar set" which is exactly the meaning of "quasieverywhere" in classical potential theory and we shall therefore use the latter terminology in this section.

If $u$ is a function from $R$ to the extended reals, $u^{(2)}=u^{\prime}$. In fact replacing $u$ by $\max \left[u, u^{\prime}\right]$ if necessary, we can suppose that $u$ is uppersemicontinuous. With this hypothesis $u=u^{\prime}$ quasieverywhere and a polar set has no fine limit points so $u$ and $u^{\prime}$ must have the same fine limit superior at every point. Specializing to indicator functions we deduce that $A^{(2)}=A^{\prime}$ : the class of fine limit points of a set is fine perfect.

Let $\Gamma$ be a family of superharmonic functions, bounded locally from below, with lower envelope $u$. Then $u$ and $u^{\prime}$ are fine upper semicontinuous, $u^{\prime} \leqq u$, and there is equality quasieverywhere. According to the fundamental convergence theorem of classical potential theory, if a function $u_{1}$ is defined as the minimum of $u$ and its limit inferior function in the ordinary topology, $u_{1}$ is superharmonic and $u=u_{1}$ 
quasieverywhere. The function $u_{1}$ is a smoothed version of $u$, and, trivially, $u^{\prime}=u_{1}^{\prime}=u_{1}$. Thus $u^{\prime}$ is identified with $u_{1}$. A corresponding identification will be stressed in the next section in the context of a general potential theory in which the analogues of superharmonic functions need not be lower semicontinuous and in which the analogous smoothing is defined differently.

Choquet [2] proved that every fine closed set is the union of a pair of sets of which the first is closed and the second has arbitrarily small capacity. [He does not state this result explicitly but it is contained in the proof of his Theorem 2 for the special case when his given set $X$ is fine closed if one replaces the first set $X_{1}$ of his decomposition by its closure.] It follows that every fine closed set is the union of an $F_{\sigma}$ set and a polar set. Using the fact that every polar set is a subset of a $G_{\delta}$ polar set it follows easily that every fine Borel set is the union of a Borel set and a polar set. Thus a completed Radon measure which vanishes on polar sets has the fine Borel sets in its domain of definition.

6. The fine topology in potential theory-probabilistic potential theory. For the background of the following work see [6], [7]. Let $R$ be a locally compact Hausdorff space with a countable topological basis and let $\left\{P_{t}, t \geqq 0\right\}$ be a semigroup of transition operators yielding, in combination with any initial distribution $\mu$, a Markov process $\left\{x_{\mu}(t)\right\}$. If $\mu$ is concentrated at the point $\xi$ we write $x_{\xi}(t)$ for $x_{\mu}(t)$. It is supposed that Hunt's condition (A) [6] is satisfied, so that the process can be chosen to have right continuous sample functions, with left hand limits at all points. A property will be said to hold $\mu$ nearly everywhere on $R$ if the set where it does not hold is a subset of an analytic set $B$ such that $B$ is hit by almost no path of the $x_{\mu}(t)$ process. (It would be no more general to suppose $B$ only nearly analytic.)

If $\xi$ is a point of $R$ and $A$ a subset of $R, A$ is said to have $\xi$ as a fine limit point if whenever $B$ is a nearly analytic superset of $A$ almost all process paths with initial point $\xi$ meet $B-B \cap\{\xi\}$ arbitrarily soon. In this way a completely regular topology, the fine topology, is defined, depending on the given family of transition operators. Concepts associated with the fine topology will be identified by the qualifier "fine." A point $\xi$ of $R$ is fine isolated if and only if almost every $x_{\xi}(t)$ path stays at $\xi$ during an initial time interval. The $\rho$ th fine limit superior function of $u$ will be denoted by $u^{(\rho)}$, and $A^{(\rho)}$ is defined correspondingly. Define $A^{+}$as the set of points regular for $A$, that is the set of points $\xi$ for which if $B$ is any nearly analytic superset of 
$A$ almost every $x_{\xi}(t)$ path meets $B$ at arbitrarily small strictly positive times. Then $A^{\prime} \subset A^{+} \subset A \cup A^{\prime}$ so if $A$ is fine closed $A^{+} \subset A$. Moreover $A^{+}$is fine closed because $\left(A^{+}\right)^{\prime} \subset\left(A \cup A^{\prime}\right)^{\prime}=A^{\prime} \subset A^{+}$. It is trivial that $\left(A_{1} \cup A_{2}\right)^{+}=A_{1}^{+} \cup A_{2}^{+}$so $\left(A \cup A^{+}\right)^{+}=A^{+} \cup A^{++} \subset A^{+} \cup A^{+}=A^{+}$. If $u$ is a function from $R$ to the extended reals define $u^{+}(\xi)$ as the supremum of the values of $c$ for which $\{\eta: u(\eta) \geqq c\}+$ contains the point $\xi$. The function $u^{+}$is fine upper semicontinuous because the set $\left\{\xi: u^{+}(\xi) \geqq c\right\}$ $=\bigcap_{n \geq 1}\{\xi: u(\xi) \geqq c-1 / n\}+$ is fine closed. Obviously $u^{\prime} \leqq u^{+}$and if there is strict inequality at a point $u^{+}=u$ at that point. If $A$ is a set, $I_{A^{+}}=\left(I_{A}\right)^{+}$. If $u$ is nearly Borel measurable, $u^{+}(\xi)=\lim \sup _{t \rightarrow 0} u\left[x_{\xi}(t)\right]$ for almost every path.

A set is called semipolar if it is a subset of a countable union $U B_{n}$ of nearly analytic sets $B_{n}$ with $B_{n}^{+}=\varnothing$. A semipolar set is thus a special fine quasinull set. Conditions true except for points of a semipolar set are commonly said to be true quasieverywhere, but we shall avoid confusion by not using this terminology.

In classical potential theory the stochastic processes are Brownian motion processes and the fine topology is that discussed in $\$ 5$. In this case $A^{\prime}=A^{+}$, the classes of fine weakly quasinull, fine quasinull, and semipolar sets coincide. A set is in this class if and only if it has no fine limit points and any member of the class is a subset of a $G_{\delta}$ set in the class. The "countable union" and "nearly analytic" in the definition of a semipolar set are pointless in this special case.

If $\lambda \geqq 0$ and if (a) $u$ is a universally measurable positive function for which $e^{-\lambda t} P_{t} u \leqq u$, the left side of the inequality increases as $t$ decreases. If (b) $\lim _{t \rightarrow 0} P_{t} u=u$, Hunt [6] called $u \lambda$-excessive and proved that $\lambda$-excessive functions are nearly Borel measurable. For each $\lambda>0$ the fine topology is the coarsest topology making $\lambda$-excessive functions continuous. For every $\mu$ a $\lambda$-excessive function is right continuous with left limits on almost every $x_{\mu}(t)$ path, and two $\lambda$ excessive functions equal on the complement of a semipolar set are identical because almost every $x_{\mu}(t)$ path meets a semipolar set in a countable parameter set.

If a function $u$ satisfies condition (a) of the preceding paragraph the function $u_{1}$ defined by

$$
u_{1}(\xi)=\lim _{t \rightarrow 0}\left(P_{t} u\right)(\xi)=\lim _{t \rightarrow 0} E\left\{u\left[x_{\xi}(t)\right]\right\}
$$

(where $E$ denotes expectation) is a commonly used smoothing of $u$. The function $u_{1}$ is $\lambda$-excessive and $u_{1} \leqq u$; hence $u_{1}^{\prime}=u_{1} \leqq u^{\prime}$.

We shall encounter a situation in which in addition to (a) it is known that $u$ is nearly Borel measurable and has a limit (necessarily 
$u^{\prime}(\xi)$ and $\left.u^{+}(\xi)\right)$ at each point $\xi$ along almost every $x_{\xi}(t)$ path, as $t \rightarrow 0$ :

$$
u^{\prime}(\xi)=u^{+}(\xi)=\lim _{t \rightarrow 0} u\left[x_{\xi}(t)\right] .
$$

Taking expectations and applying Fatou's lemma,

$$
u^{\prime}(\xi)=u^{+}(\xi)=E\left\{\lim _{t \rightarrow 0} u\left[x_{\xi}(t)\right]\right\} \leqq u_{1}(\xi)
$$

so that $u^{\prime}=u^{+}=u_{1} \leqq u$ in this situation. Moreover the set where $u>u^{\prime}$ is not only fine quasinull but even semipolar. In fact this set is a countable union of sets of the form

$$
\left\{\xi: u(\xi) \geqq b>a \geqq u^{\prime}(\xi)\right\} .
$$

Each of these sets is a nearly measurable Borel set $A$, with $A^{+}=\varnothing$ because if $\xi$ were a point in $A^{+}$it would satisfy $u^{+}(\xi) \geqq b, a \geqq u^{\prime}(\xi)$ $=u^{+}(\xi)$.

We shall frequently impose Meyer's condition on the transition probabilities:

(L) There is a Radon measure $\mu$ on the space $R$, with $\mu(R)=1$, such that $\mu(A)>0$ whenever $A$ is a nearly analytic fine open set.

The normalization to a probability measure is of course unessential but will be convenient below. Meyer pointed out [7] that this condition is equivalent to the condition that for some $\lambda>0$ (equivalently every $\lambda>0$ ) there be a Radon measure $m$ such that if $u$ is $\lambda$-excessive and vanishes $m$ almost everywhere then $u$ vanishes identically. In the classical case this condition is satisfied with $m$ supported by any singleton. We shall sometimes impose a further condition, also satisfied in the classical case:

$\left(\mathrm{L}^{\prime}\right)$ For every $\xi$ in $R$, almost no $x_{\xi}(t)$ path meets $\xi$ for $t$ strictly positive but sufficiently small unless the path stays at $\xi$ during an initial time interval, that s, unless $\xi$ is a fine isolated point of $R$.

The condition $\left(\mathrm{L}^{\prime}\right)$ is equivalent to the condition that $A^{+}=A^{\prime}$ for every $A$.

Parts (i) (due to the writer) and (iii) (due to Meyer) of the following theorem are not new but the proofs are sketched to illustrate the point of view of this paper. Part (ii) may be useful when it is desirable to avoid hypothesis (L).

THEOREM 6.1. Let $u$ be the lower envelope of a family of $\lambda$-excessive functions.

(i) If the family is countably infinite, $u^{\prime}$ is $\lambda$-excessive, $u^{\prime} \leqq u$, and there is equality except on a semipolar set. Moreover for every $\xi u$ has 
$u^{\prime}(\xi)=u^{+}(\xi)$ as limit at $\xi$ in the fine sense and on almost every $x_{\xi}(t)$ path $(t \rightarrow 0)$.

(ii) In the general case to each probability distribution $\mu$ on $R$ corresponds the lower envelope ${ }_{\mu} u$ of some countable subfamily such that ${ }_{\mu} u^{\prime} \geqq u$ except on a semipolar set and, for every $v$ in the family ${ }_{\mu} u^{\prime} \leqq v$ $\mu$ nearly everywhere on $R$.

(iii) In the general case under hypothesis $(\mathrm{L}) u^{\prime}$ is $\lambda$-excessive and there is a countable subfamily with lower envelope $u_{\infty}$ such that $u_{\infty}^{\prime}=u_{\infty}^{+}$ $=u^{\prime}=u^{+} \leqq u \leqq u_{\infty}$, with equality except on a semipolar set.

We can suppose that the given family is uniformly bounded.

Proof of (i). If $u$ is the lower envelope of a countable family of $\lambda$-excessive functions $u$ is fine upper semicontinuous, nearly Borel measurable, and $e^{-\lambda t} P_{t} u \leqq u$. Thus $u$ satisfies condition (a) of the two conditions defining $\lambda$-excessive functions. Hence $u^{\prime}$ is $\lambda$-excessive. The standard upcrossing inequalities show that for $0 \leqq r_{1}<r_{2}$ the expected number of upcrossings of $\left[r_{1}, r_{2}\right]$ by $v\left[x_{\mu}(\cdot)\right]$ is bounded independently of the member $v$ of the given family, from which it follows that almost no sample function of the $u\left[x_{\mu}(t)\right]$ process has an oscillatory discontinuity. In particular if $\mu$ is supported by the singleton $\{\xi\} u$ must have a limit at $\xi$ along almost every path of the $x_{\xi}(t)$ process as $t \rightarrow 0$. We have seen in the preliminary discussion at the beginning of this section that then the conclusions of (i) are true.

Proof of (ii). Let $S$ be a countable dense set of parameter values and choose a countable subfamily of the given family of $\lambda$-excessive functions in such a way that the lower envelope ${ }_{\mu} u$ of the subfamily satisfies

$$
{ }_{\mu} u\left[x_{\mu}(t)\right]=\operatorname{ess} \inf v\left[x_{\mu}(t)\right], \quad t \in S,
$$

where $v$ ranges through the entire family. Applying (i), ${ }_{\mu} u^{\prime}$ is $\lambda$ excessive and if $v$ is in the family

$$
{ }_{\mu} u^{\prime}\left[x_{\mu}(t)\right] \leqq v\left[x_{\mu}(t)\right], \quad t \in S
$$

with probability 1 . Since ${ }_{\mu} u^{\prime}$ and $v$ are right continuous as functions of the parameter on almost all sample paths ${ }_{\mu} u^{\prime} \leqq v \mu$ nearly everywhere on $R$. On the other hand ${ }_{\mu} u \geqq u$ and ${ }_{\mu} u^{\prime}={ }_{\mu} u$ off a semipolar set. Hence ${ }_{\mu} u^{\prime} \geqq u$ off a semipolar set.

Proof of (iii). Under (L) take $\mu$ in (ii) as the measure whose existence is supposed in (L). Then the fact that ${ }_{\mu} u^{\prime} \leqq v \mu$ nearly everywhere and that both sides are fine continuous implies that the inequality holds everywhere. Hence ${ }_{\mu} u^{\prime} \leqq u$ so ${ }_{\mu} u^{\prime} \leqq u \leqq{ }_{\mu} u$. Taking primes in this inequality yields $u^{\prime} \leqq{ }_{\mu} u^{\prime}$. Thus (iii) is true with $u_{\infty}={ }_{\mu} u$. 
In probabilistic potential theory connections between the given topology of $R$ and the fine topology play an essential role. One such connection, linked with the complete regularity of the fine topology, is the fact that the fine topology is the coarsest making the $\lambda$-excessive functions continuous, if $\lambda>0$. A more specific result than this was proved by Hunt [6] who showed that if $A$ is a nearly analytic set and if $\xi$ is a point not in $A \cup A^{+}$then if $\lambda>0$ there is a $\lambda$-excessive function equal to 1 on $A$ and less than 1 at $\xi$. According to the definition of $A^{+}$the same result holds if $A$ is arbitrary. Hunt's result was, more specifically, the following. Let $V(A)$ be the $\lambda$ probability (probability computed using $e^{-\lambda t} P_{t}$ instead of $\left.P_{t}\right)$ that an $x_{\xi}(t)$ path hits the nearly analytic set $A$ at some strictly positive time. If $A$ is an arbitrary set let $U(A)$ be the lower envelope of the $\lambda$-excessive functions $\geqq 1$ on $A$. Hunt proved that if $A$ is nearly analytic $V(A)$ is $\lambda$ excessive, $V(A) \leqq U(A)$, and there is equality except on $A-A \cap A^{+}$, where, trivially, $V(A)<1=U(A)$.

The additional connections between the given and fine topologies listed in the following lemmas are mostly known, at least in their set versions, but are given for ready reference and because they are not otherwise conveniently available. We continue to use the notation $U(A)$ and $V(A)$ as just defined, supposing that $\lambda$ is some strictly positive number fixed throughout the discussion.

Lemma 6.1. Suppose that (L) is true.

(i) If $A$ is nearly analytic, $U(A)^{\prime}=U(A)^{+}=V(A)$.

(ii) If $A$ is fine closed, $A^{+}$is the set where $U(A)^{\prime}=1$.

(iii) If $u$ is an arbitrary function from $R$ to the extended reals $u^{+}$is nearly Borel measurable and the set where $u>u^{+}$is semipolar. In particular if $u \equiv u^{+}$(and this is true if $\left.u \equiv u^{\prime}\right) u$ is nearly Borel measurable.

Proof of (i). If $A$ is nearly analytic we have already noted Hunt's theorem that $V(A) \leqq U(A)$ with equality except on $A-A \cap A^{+}$so taking primes and applying Theorem 6.1 we obtain

$$
V(A) \leqq U(A)^{\prime}=U(A)^{+} \leqq U(A)
$$

and there is equality except perhaps on $A-A \cap A^{+}$. If $\xi$ is in this set almost every $x_{\xi}(t)$ path misses $A$ during an initial open time interval, so there is equality above on the part of the path corresponding to this interval. But then since $\lambda$-excessive functions are right continuous on almost all $x_{\xi}(t)$ paths, $V(A)(\xi)=U(A)^{\prime}(\xi)$, as was to be proved.

Proof of (ii). Let $A$ be a fine closed set. Applying Hunt's theorem, $A$ is the set where $U(A)=1$. If $\xi$ is in $A^{+}, U(A)^{\prime}(\xi)=1$ because $U(A)$ has the limit $U(A)^{\prime}(\xi)$ on almost every $x_{\xi}(t)$ path when $t \rightarrow 0$. Con- 
versely if $\xi$ is not in $A^{+}$there is a nearly analytic superset $B$ of $A$ such that almost no $x_{\xi}(t)$ path meets $B$ in some initial open time interval. That is, $V(B)(\xi)<1$. But then $U(A)^{\prime}(\xi) \leqq U(B)^{\prime}(\xi)=V(B)(\xi)<1$, as was to be proved.

Proof of (iii). If $A$ is fine closed (ii) implies that $A^{+}$is nearly Borel measurable. For an arbitrary set $A, A \cup A^{+}$is fine closed and $A^{+}=\left(A \cup A^{+}\right)^{+}$so $A^{+}$is nearly Borel measurable. For any function $u$ from $R$ to the extended reals $u^{+}$is nearly Borel measurable because

$$
\left\{\xi: u^{+}(\xi) \geqq c\right\}=\bigcap_{n \geqq 1}\{\xi: u(\xi) \geqq c-1 / n\}+
$$

If $A$ is fine closed $A-A^{+}$is semipolar because it is a subset of the set where $U(A)>U(A)^{\prime}$. More generally for any set $B$ the set $B-B \cap B^{+}$ is semipolar because this difference is $A-A^{+}$where $A$ is the fine closed set $B \cup B^{+}$. Still more generally, if $u$ is any function from $R$ to the extended reals, the set where $u>u^{+}$is semipolar because if $\eta$ is in the set $C^{+}$, where $C=\left\{\xi: u(\xi) \geqq b>a \geqq u^{+}(\xi)\right\}$, then $u^{+}(\eta) \geqq b$ so $C \cap C^{+}=\varnothing$. Hence $C=C-C \cap C^{+}$is semipolar.

Lemмa 6.2. Suppose that (L) is true.

(i) Every fine quasinull [semipolar] set $A$ is a subset of a union $\cup B_{n}$ with $B_{n}$ nearly Borel measurable and $B_{n}^{\prime}=\varnothing\left[B_{n}^{+}=\varnothing\right]$

(ii) If ( $\left.\mathrm{L}^{\prime}\right)$ is also true every fine weakly quasinull set is semipolar.

Proof of (i). It is sufficient to prove that (i) is true for any set $A$ with $A^{\prime}=\varnothing\left[A^{+}=\varnothing\right]$. Since $A$ is the union of the fine closed nearly Borel measurable set $A^{+}$and a subset of the set where $U(A)>U(A)^{\prime}$ it need only be shown that the latter set is a subset of a countable union of nearly Borel measurable sets $B_{n}$ with $B_{n}^{+}=\varnothing$. By Theorem 6.1 there is a countable lower envelope $U_{\infty}(A)$ of the class defining $U(A)$ such that $U_{\infty}(A) \geqq U(A)$ and $U_{\infty}(A)^{\prime}=U(A)^{\prime}$. Since $U_{\infty}(A)$ is fine upper semicontinuous and nearly Borel measurable, we need only remark that the set where $U_{\infty}(A)>U_{\infty}(A)^{\prime}$ is a countable union of sets of the form $\left\{\xi: U_{\infty}(A)(\xi) \geqq b>a \geqq U_{\infty}(A)^{\prime}(\xi)\right\}$ and that each of these sets is nullified by the plus operation.

Proof of (ii). Since every fine weakly quasinull set is a countable union of sets of the form $A-A^{\prime}$ where $A$ is fine closed, and since under $\left(\mathrm{L}^{\prime}\right) A^{\prime}=A^{+}$(ii) is a consequence of Lemma 6.1 (iii) as applied to the indicator function of $A$.

LEMмa 6.3. Suppose that (L) is true. If $u$ is any function from $R$ to the extended reals there is a countable ordinal $\sigma$ such that $u^{(\sigma)}=u^{(\sigma+1)}$

The set version of Lemma 6.3 states that for any set $A$ there is a 
countable ordinal $\sigma$ such that $A^{(\sigma)}=A^{(\sigma+1)}$. The truth of this version implies that of the general case. In fact the set version implies that there is a countable ordinal $\sigma$ so large that if $r$ is rational $\{\xi: u(\xi) \geqq r\}^{(\rho)}$ $=B_{r}$ is independent of $\rho \geqq \sigma$. Then by (2.1) $B_{r} \subset\left\{\xi: u^{(\rho)}(\xi) \geqq r\right\} \subset B_{s}$ for $\rho \geqq \sigma$ and $s<r$. If $B_{t-}=\bigcap_{s<t} B_{s}$ this means that $\left\{\xi: u^{(\rho)}(\xi) \geqq t\right\}$ $=B_{t-}$ for all real positive $t$ when $\rho \geqq \sigma$, so $u^{(\sigma)}=u^{(\sigma+1)}$ as was to be proved. To prove the set version of the lemma let $\lambda$ be strictly positive and let $A$ be a fine closed set. Then $A^{(\rho)}$ is the set where $U\left(A^{(\rho)}\right)$ $=1$ so we need only show that $U\left(A^{(\rho)}\right)$ does not depend on $\rho$ for large $\rho$. Now $U\left(A^{(\rho)}\right)^{\prime}$ is $\lambda$-excessive and

$$
U\left(A^{(\rho+2)}\right)^{\prime} \leqq U\left(A^{(\rho+2)}\right) \leqq U\left(A^{(\rho+1)}\right)^{\prime} \leqq U\left(A^{(\rho+1)}\right) \leqq U\left(A^{(\rho)}\right)^{\prime} .
$$

By Theorem 6.1 (iii) there is a countable subclass of $\left\{U\left(A^{(\rho)}\right)^{\prime}\right\}$ whose lower envelope $v$ has the property that $v^{\prime} \leqq U\left(A^{(\rho)}\right)^{\prime}$ for all $\rho$. This means that there is an ordinal $\sigma$ such that $U\left(A^{(\rho)}\right)^{\prime}=U\left(A^{(\sigma)}\right)^{\prime}$ for $\rho>\sigma$ and the displayed inequality above shows that then $U\left(A^{(\rho)}\right)$ does not depend on $\rho$ for large $\rho$, as was to be proved.

Under (L) if $A$ is fine closed we have shown that $A=A_{1} \cup A_{2}$ where $A_{1}\left(=A^{+}\right)$is nearly Borel measurable and $A_{2}$ is semipolar. If $A$ is an arbitrary set which can be expressed in this way the fact that $A_{2}$ is a subset of a nearly Borel semipolar set $B$ which can be assumed not to meet $A_{1}$ yields the fact that (denoting complementation by $\sim$ )

$$
\tilde{A}=\left(A_{1} \cup B\right) \sim\left(B-A_{2}\right)
$$

so that $\tilde{A}$ is also the union of a nearly Borel measurable set and a semipolar set. From this we conclude that every fine Borel set is the union of a nearly Borel measurable set and a semipolar set and more generally that every fine Borel measurable function is equal off a semipolar set to some nearly Borel measurable function. Since nearly Borel measurable sets are universally measurable, if $\mu$ is a completed Radon measure which vanishes on semipolar sets what we have proved implies that, under $(\mathrm{L}), \mu$ is defined on the class of fine Borel sets. Such a measure is a [weak] quasicapacity if it even vanishes on fine [weakly] quasinull sets. If $\left(\mathrm{L}^{\prime}\right)$ is also satisfied, fine weakly quasinull sets are semipolar, so any $\mu$ vanishing on semipolar sets is now a fine quasicapacity and fine weak quasicapacity.

Getoor pointed out in a letter that a modification of Hunt's proof [6] that excessive functions are nearly Borel measurable shows that under (L) excessive functions are even Borel measurable. The proof of Lemma 6.1 can be sharpened using this fact to yield the result that under $(\mathrm{L})$ if $u$ is an arbitrary function from $R$ to the extended reals $u^{+}$is Borel measurable. The function $v$ in Theorem 8.2 , the set $F$ in Theorem 8.3, and the function $u$ in Theorem 8.4 below are therefore 
Borel measurable. In Lemma 6.2 (i) the set $B_{n}$ can be supposed Borel measurable. The preceding paragraph can be strengthened: under (L) every fine Borel measurable function is equal off a semipolar set to some Borel measurable function.

7. Application to classical potential theory. We use the notation and conventions of $\$ 5$ in this section.

TheOREM 7.1. The space of classical potential theory, with the fine topology has the quasiLindelöf $f^{(1)}$ property.

Since the classes of fine quasinull and weakly quasinull sets are the same in classical potential theory it is sufficient to prove the theorem using the criterion of Lemma 2.1 for the weak quasiLindelöf ${ }^{(1)}$ property. The proof of Theorem 4.1 can be carried over to this theorem almost word for word. Let $\Gamma=\Gamma_{0}$ be a family of fine closed sets with intersection $F$. In applying Lemma 2.1 it is no restriction to assume that $\Gamma$ contains the countable intersections of its member sets. We shall apply the set version of Theorem 3.1 to show that a set $F_{\infty}$ exists as described in Lemma 2.1 with $n=1$. We use the set versions of axioms $\boldsymbol{A}_{1}, \boldsymbol{A}_{2}, \boldsymbol{A}_{3}$, defining $A^{*}=A^{\prime}$. The first two axioms are obviously satisfied. Let $X$ be the set of extended real numbers with the usual order and $G$ a finite union of sets of a countable basis for the topology of $R$. If $A$ is fine closed define $C_{G}(A)$ as the capacity of $G \cap A$. We are using Choquet's theorem that a fine closed set is the union of an $F_{\sigma}$ set in the given topology and a polar set, so that $G \cap A$ is capacitable. If $\boldsymbol{C}_{G}(A) \leqq \boldsymbol{C}_{G}(B)$ for every $G$ we shall show below that $A \subset B$ neglecting a polar set, so $A^{\prime} \subset B^{\prime}$. Thus the hypotheses of the set version of Theorem 3.1 are satisfied, with $\boldsymbol{C}(A)=\left\{\boldsymbol{C}_{G}(A)\right\}$, as was to be proved. (Just as in the proof of Theorem 4.1 the criterion of Theorem 2.1 can be used instead of that of Lemma 2.1, in fact with the same choice of $X$ as in Theorem 4.1, and with $\nu$ replaced by the capacity function.) There remains the proof that $\mathbf{C}_{G}(A) \leqq \mathbf{C}_{G}(B)$ for all $G$ in the indicated class implies that $A \subset B$ neglecting a polar set. It is elementary that the given inequality must hold for all open $G$. One can then prove the desired result by applying the Wiener criterion that a point be a fine limit point of a set to show that $A^{\prime} \subset B^{\prime}$. Alternatively one can extend the given equality to all compact $G$ and thereby find that if $G$ is any compact subset of $A-A \cap B$ the capacity of $G$ vanishes, and this fact implies the desired result. This proof of Theorem 7.1 is given for comparison with the proof of Theorem 4.1. The proof of Theorem 8.1 
uses a different choice of $X$ and $C$ which is more elegant and is of course applicable to the present special case.

Theorem 2.2 is applicable to ordinary capacity, since the latter is a fine quasicapacity and weak quasicapacity in the sense of $\$ 2$. To apply Theorems 2.3 and 2.4 the proper hypothesis on $\mu$ is that $\mu$ be a completed Radon measure in the original topology, vanishing on polar sets. We have pointed out that such a measure is defined on the class of fine Borel sets so that Theorems 2.3 and 2.4 are applicable in the present context.

Getoor [5] proved in a probabilistic context a theorem (see \$8) which implies that in classical potential theory a Radon measure vanishing on polar sets has a smallest fine closed support. Choquet [2] then gave a nonprobabilistic proof of this result, which in our development is an application of Theorem 2.3 to the fine topology. Brelot [1] showed that various functionals in a potential theoretic context including the classical case are what we have called in \$2 quasicapacities (fine topology) and applied the methods of an unpublished version of the present paper to these functionals.

8. Application to probabilistic potential theory. We use the notation of $\$ 6$.

TheOREM 8.1. The space of probabilistic potential theory, in the fine topology, under condition (L), has the quasiLindelöf(1) property. More specifically, given a family of fine upper semicontinuous functions with lower envelope $u$ there is a countable subfamily with lower envelope $u_{\infty}$ such that $u_{\infty}^{+} \leqq u \leqq u_{\infty}$.

Note that $u_{\infty}=u_{\infty}^{+}$fine quasieverywhere, and in fact except on a semipolar set, and that $u^{\prime} \leqq u_{\infty}^{+}$so that the result proved is definitely stronger than that the space has the fine quasiLindelö ${ }^{(1)}$ property, unless $\left(L^{\prime}\right)$ is satisfied when the two properties are equivalent.

We shall prove the theorem in its set version, in which the functions involved are indicator functions of fine closed sets. A trivial modification of the argument in the proof of Theorem 2.1 (replacing ${ }^{(n)}$ by ${ }^{+}$) shows that this is sufficient. Suppose then that $\Gamma=\Gamma_{0}$ is a family of fine closed sets with intersection $F$. We shall apply Theorem 3.1 to show that a countable intersection $F_{\infty}$ of sets in $\Gamma$ exists, satisfying $F_{\infty}^{+} \subset F \subset F_{\infty}$. It is no restriction to assume in the proof that $\Gamma$ already contains the countable intersections of its members. We use the set version of Axioms $A_{1}, A_{2}, A_{3}$ in $\$ 3$, defining $A^{*}=A^{+}$. Fix $\lambda>0$ and let $X$ be the space of $\lambda$-excessive functions, ordered by pointwise inequality. Then every subset of $X$ has a countable infimum by 
Theorem 6.1 (iii). Define $\mathrm{C}(A)=U(A)^{\prime}$. Then $A^{+}$is the set where $\mathbf{C}(A)=1$. Hence $\mathbf{C}(A) \leqq \mathbf{C}(B)$ implies that $A^{+} \subset B^{+}$and Theorem 3.1 is applicable to yield the existence of the desired set $F_{\infty}$.

As we remarked in $\$ 2$ the validity of the quasiLindelöf property (in the fine topology) implies that every (fine) weakly quasinull set is (fine) quasinull. Thus we need not distinguish between these types of sets in the following. A trivial covering argument shows that every fine quasinull set is the union of a countable set and a semipolar set. The fine quasinull sets are thus semipolar if and only if singletons not corresponding to fine isolated points are semipolar, that is, if and only if $\left(\mathrm{L}^{\prime}\right)$ is satisfied.

Theorem 8.2. In the space of probabilistic potential theory, under condition (L), if $u$ is a fine upper semicontinuous function there is a unique function $v$ such that (a) $v$ is fine upper semicontinuous, (b) $v \leqq u$, with equality off a semipolar [fine quasinull] set and (c) if $v_{1}$ satisfies (a) and (b) $v_{1} \geqq v$. The function $v$ is nearly Borel measurable and $v=v^{+}\left[v=v^{\prime}\right]$.

Let $v$ be the lower envelope of the class of functions satisfying (a) and (unbracketed) (b). According to Theorem 8.1 there is a countable lower envelope $v_{\infty}$ of members of this class such that $v_{\infty}^{+} \leqq v \leqq v_{\infty}$. Since $v_{\infty}^{+}$is in the class we conclude that $v_{\infty}^{+}=v$. Thus $v$ is in the class, $v=v^{+}$ and $v$ is nearly Borel measurable, as was to be proved. The bracketed assertion is proved similarly.

We have seen in $\$ 6$ that under (L) if $\mu$ is a completed Radon measure in terms of the given topology of $R$ and if this measure vanishes on semipolar sets then the measure is defined on the class of Borel sets.

TheOREM 8.3. Under (L), if $\mu$ is a completed Radon measure, vanishing on semipolar sets, $\mu$ has a smallest fine closed support $F$, with $F=F^{+}$.

Under $\left(\mathrm{L}^{\prime}\right)$ the classes of fine weakly quasinull, fine quasinull and semipolar sets are identical, so this theorem becomes just an application of Theorem 2.3. Without $\left(\mathrm{L}^{\prime}\right)$ the proof of Theorem 2.3 is applicable in the present context since, under (L) (in the notation of that proof) $F_{\infty}-F$ is semipolar, and $A^{+}$is a fine closed support of $\mu$ if $A$ is.

Theorem 2.4 corresponds to a result generalizing Theorem 8.3.

THEOREM 8.4. Let $f_{1}, f_{2}$ be functions from $R$ to the extended reals with $f_{1} \leqq f_{1}^{+}$and let $\mathfrak{N}$ be a class of subsets of $R$ containing the semipolar sets and containing the countable unions of its members. Then under (L) 
the lower envelope $u$ of the class of fine upper semicontinuous functions $\geqq f_{1}$ on $R$ and also $\geqq f_{2}$ neglecting a set in $\mathfrak{N}$ is in the class, and $u=u^{+}$.

The proof of Theorem 2.4 is applicable if "' is replaced by "+". Theorem 8.4 becomes a specialization of Theorem 2.4 if $\left(\mathrm{L}^{\prime}\right)$ is satisfied.

For any initial distribution $\mu$ there is a corresponding stochastic process $\left\{x_{\mu}(t), t \geqq 0\right\}$. Here $x_{\mu}(t)$ is a random variable with value $x_{\mu}(t, \omega)$ at the point $\omega$ of a certain measure space with measure $P_{\mu}$. Suppose that $(t, \omega) \rightarrow \alpha(t, \omega)$ is an extended real valued function of the pair $(t, \omega)$. Suppose that $\alpha(\cdot, \omega)$ is continuous and monotone increasing on $[0, \infty)$ for fixed $\omega$ and that $\alpha(t, \cdot)$ is $P_{\mu}$ measurable for fixed $t$, for every $\mu$. If $A$ is a nearly analytic set and if $\omega$ is fixed, the set of values of $t$ for which $x_{\mu}(t, \omega) \in A$ may be a support of the measure $d_{t} \alpha(t, \omega)$ for $P_{\mu}$ almost all $\omega$. If this is true simultaneously for every $\mu$, $A$ is said to be a support for $\alpha$. If $A$ is semipolar (in which case, under (L) $A$ is a subset of a nearly Borel semipolar set), the $d_{t} \alpha(t, \omega)$ measure of the set of values of $t$ in question is 0 for $P_{\mu}$ almost every $\omega$ because almost every $x_{\mu}(t)$ path meets $A$ at a countable set of parameter values. Since every fine closed set differs from a nearly Borel set by a semipolar set, if $A$ is fine closed and $\omega$ is fixed the set of values of $t$ in question is a Borel set, if an $\omega$ set of $P_{\mu}$ measure 0 is excluded, so the concept of a fine closed support of $\alpha$ is now meaningful.

If $\alpha$ is a continuous additive functional Getoor [5] showed (even without assuming (L)) that the functional has a smallest fine closed support. He pointed out (orally-for the details see Meyer [8]) that the existence of this support implies, under hypotheses much stronger than (L), hypotheses which imply the existence of a certain correspondence between continuous additive functionals and measures which vanish on semipolar sets, that a measure of the latter type has a smallest fine closed support. Theorem 8.3 is thus Getoor's measure result with weaker hypotheses. We now go in the reverse direction and, assuming only (L), prove using Theorem 8.3 that any function $\alpha$, satisfying the hypotheses of the preceding paragraph but not necessarily a continuous additive functional, has a smallest fine closed support.

THEOREM 8.5. If $\alpha$ is as described then (under (L)) $\alpha$ has a smallest fine closed support $F ; F=F^{+}$and $F$ is nearly Borel measurable.

The fact that $F=F^{+}$implies that $F$ is nearly Borel measurable, according to Lemma 6.1. We can suppose that $\alpha$ is bounded, replacing $\alpha$ by $\arctan \alpha$ if necessary to achieve boundedness. For every $\mu$, 
$\alpha$ is measurable with respect to the product of the field of Borel $t$ sets and the field of $P_{\mu}$ measurable $\omega$ sets. For each $\mu$ and Borel subset $A$ of $R$ define $\nu_{\mu}(A)$ as the $P_{\mu}$ integral of the $d_{t} \alpha(t, \omega)$ measure of the set where $x_{\mu}(t, \omega) \in A$. Then $\nu_{\mu}$ is a measure, and we shall assume that it has been completed. If $A$ is nearly Borel measurable it is obviously in the domain of $\nu_{\mu}$, and if $A$ is nearly Borel measurable and semipolar $\nu_{\mu}(A)=0$ since almost every $x_{\mu}(t)$ path hits $A$ in a countable parameter set. If $A$ is an arbitrary semipolar set it is a subset of a nearly Borel measurable semipolar set so again $\nu_{\mu}(A)=0$. Thus the measure $\nu_{\mu}$ is a completed Radon measure vanishing on semipolar sets. As such we have seen that this measure is defined on the class of fine Borel sets. The set $A$ is a support for every $\nu_{\mu}$ if and only if $A$ is a support of $\alpha$. Now according to the trivial generalization of Theorem 2.3 noted after the proof of that theorem, translated into the context of Theorem 8.3, the class of measures $\left\{\nu_{\mu}\right\}$ has a smallest common fine closed support. That is, there is a fine closed set $F$ which is a support of $\nu_{\mu}$ for every $\mu$ and which is the smallest fine closed set with this property. This set is the smallest fine closed support of $\alpha$, and $F=F^{+}$according to (our extension of) Theorem 8.3.

\section{BIBLIOGRAPHY}

1. M. Brelot, Capacity and balayage for decreasing sets, Proc. Fifth Berkeley Symposium (to appear).

2. Gustave Choquet, Sur les points d'effilement d'un ensemble, Application à l'étude de la capacite, Ann. Inst. Fourier (Grenoble) 9 (1959), 91-101.

3. - Demonstration non probabiliste d'un theorème de Getoor, Ann. Inst. Fourier 15 (1965), 409-414.

4. Casper Goffman, C. J. Neugebauer and T. Nishiura, Density topology and approximate continuity, Duke Math. J. 28 (1961), 497-505. 1964.

5. R. K. Getoor, Additive functionals of a Markov process, Lectures at Hamburg,

6. G. A. Hunt, Markoff processes and potentials. I, Illinois J. Math. 1 (1957), 44-93.

7. P. A. Meyer, Fonctionelles multiplicatives et additives de Markov, Ann. Inst Fourier (Grenoble) 12 (1962), 125-230.

8. - Le support d'une fonctionelle additive continue, Séminaire BrelotChoquet-Deny, no. 10, $9(1964 / 65), 12$ pp.

9. A. P. Morse, Perfect blankets, Trans. Amer. Math. Soc. 61 (1947), 418-442.

10. Stanislaw Saks, Theory of the integral, Monografie Matematyczne, vol. 7, Warsaw-Lwow, 1937.

11. Robert E. Zink, On semicontinuous functions and Baire functions, Trans. Amer. Math. Soc. 117 (1965), 1-9. 